\title{
Theories of Immunosurveillance/Immunoevasion: An attempt to explain Persistence of Cancer
}

\author{
Kirtimaan Syal* and Dibyajyoti Banerjee \\ Department of Experimental Medicine and Biotechnology, \\ Post Graduate Institute of Medical Education and Research, Chandigarh 160012
}

Immunosurveillance theory advocates that cancer frequently arises in the body, recognized as foreign and eliminated by the immune system. It was first conceptualized by Paul Ehrlich in 1900s. After around 50 years, Lewis Thomas laid the building block of immunosurveillance by hypothesizing that cell mediated branch of immune system has evolved to locate cancerous cells and kill them. In 1950s and early 1960, it has been realized that the immune system could recognize and respond to tumor-associated antigens ${ }^{1}$. Thus, supporting the theory of immunosurveillance.

But if there is a surveillance system than why does cancer exist???

In 1974, it has been reported that nude mice do not demonstrate an increase in tumor incidence thus contradicting the hypothesis of tumor immunosurveillance ${ }^{2}$. However, subsequent observations that nude mice do not completely lack functional $\mathrm{T}$ cells and those two components of the immune system IFNg and perforin help to prevent tumour formation in mice have led to renewed interest in a tumour-suppressor role for the immune respons $\mathrm{e}^{3-4}$. Many scientists believe that cancer occurs because of the lack of these surveillance mechanisms and immunoevasion have no role to play. But, recent research reveals about the potential of cancerous cells in evading immune system via mechanisms of immunoevasion. So there are two possible ways via which cancerous cells can be persistent in the human body, one describes the inability of our immune system to respond well to these cancer cells (due to defect in immunity/immunosurveillance) and the other possibility advocates the evasion of immune cells by cancer cells (i.e. immunoevasion).

Defects in immunosurveillance mechanisms involves defective $\mathrm{T}$ cell anergy, the existence of more number of regulatory $\mathrm{T}$ cells, systemic defects of dendritic cells derived from tumor patients, secretion of immunosuppressive cytokines, resistance to apoptosis, and deficient expression of immunomodulatory molecules. Immunoevasion mechanisms include masking of MHCs and lack of co-stimulatory signals.

Patients who have undergone transplantation take immunosuppressive drugs. Increased incidence of skin cancer and lymphomas in such patients well demonstrates the role of immunosurveillance. In older patients and long surviving grafts, $44 \%$ cases of skin cancer and $14 \%$ of other cancer have been reported ${ }^{5}$. On the other hand, capacity of cancer cells to hide the MHCs on their confrontation with immune cell, demonstrate the mechanism of immunoevasion.

Schreiber group reported the increase in incidence of chemically induced tumors having defective P53 suppressor gene, due to which they are resistant to apoptosis4. Mark Smyth group illustrated the perforin mediated killing as an important mechanism in tumor immunity ${ }^{6-7}$. Recently number of endogenous molecules that are hypothesized to be produced by cancer cells and acting as the ligand of toll like receptor, have been reported to be contaminated by Pathogen associated microbial pattern molecules which are acting as an artifact, thus supporting the theory of immune surveillance. On the other hand, change in the level of growth receptors and omission of the receptors complementary to immune cells supports the hypothesis of immunoevasion.

AIDS, the condition in which T helper cells count goes below 200 and patient become susceptible to various malignancies like Kaposi sarcoma and cervical cancer illustrates the significance of immunosurveillance. In humans, there are 100 trillion cells which include more than 1000 different types. But still very low frequency of cancer is observed. Why??? It could be explained on the basis of immunosurveillance.

Further genetic susceptibility for cancer describes the inbuilt genetic defects in surveillance. Thus, explaining the persistence of cancer cells to be dependent on defects in immuno surveillance. Tumor patients with higher titre of tumor infiltrating lymphocytes showed a survival rate of $38 \%$ as compared to only $4.5 \%$ for patients lacking tumor infiltrating lymphocytes ${ }^{8}$. It has been shown that people having large number of tumor infiltrating lymphocytes have significantly lower incidence of metastasis. Based on this concept, adoptive immunotherapy has been supervised. 
One of the other important phenomenons is spontaneous resolution. Tumor occurs but in most of the cases they are cured themselves. Clinical evidences related to self curing of hepatoblastomas and retinoblastomas are well illustrated ${ }^{9-10}$. And the count of self curing in tumors is very large. In fact most of the cases of spontaneous resolution are never accessible to clinicians.

But, many reports suggest the masking of tumor specific antigens and tumor associated antigens on confrontation with the immune cell. Tumor cells produce cytokines and receptors essential for replication and spread. These facts strongly advocate the theory of tumor evasion.

We can conclude that both tumor surveillance and tumor evasion are essential for persistence of cancer. Initially, scientific community advocated immunosurveillance, but now research supports immunoevasion as well.

\section{References:}

1. Old LJ, Boyse EA. Immunology of Experimental Tumors. Annu Rev Med. 1964;15:167-186.

2. Stutman O. Tumor development after 3-methylcholanthrene in immunologically deficient athymic-nude mice. Science. Feb 8 1974;183(124):534-536.

3. Shankaran V, Ikeda H, Bruce AT, et al. IFNgamma and lymphocytes prevent primary tumour development and shape tumour immunogenicity. Nature. Apr 26 2001;410(6832):1107-1111.

4. Kaplan DH, Shankaran V, Dighe AS, et al. Demonstration of an interferon gamma-dependent tumor surveillance system in immunocompetent mice. Proc Natl Acad Sci U S A. Jun 23 1998;95(13):7556-7561.

5. MacLeod AM, Catto GR. Cancer after transplantation. BMJ. Jul 2 1988;297(6640):4-5.

6. van den Broek ME, Kagi D, Ossendorp F, et al. Decreased tumor surveillance in perforin-deficient mice. J Exp Med. Nov 1 1996;184(5):1781-1790.

7. Street SE, Trapani JA, MacGregor D, Smyth MJ. Suppression of lymphoma and epithelial malignancies effected by interferon gamma. J Exp Med. Jul 1 2002;196(1):129-134.

8. Zhang L, Conejo-Garcia JR, Katsaros D, et al. Intratumoral T Cells, Recurrence, and Survival in Epithelial Ovarian Cancer. New England Journal of Medicine. 2003;348(3):203-213.

9. Abbey AM, Flores RM. Spontaneous resolution of a pericardial cyst. Ann Thorac Cardiovasc Surg. Aug 2010;16(1):55-56.

10. McPherson T, Ogg G. Spontaneous resolution of basal cell carcinoma in naevoid basal cell carcinoma syndrome/Gorlin's syndrome. Clin Exp Dermatol. Dec 2009;34(8):e884-885. 